\title{
Psychometric Evaluation of the Dietary-Adherence Intake and Eating Test (DIET): A Measure for Early Detection of Maladaptive Eating Behaviors after Bariatric \\ Surgery
}

Keywords: Bariatric surgery; Post-bariatric dietary adherence: Post-bariatric assessment; Maladaptive eating behaviors; Disordered eating; Suboptimal weight loss outcomes

\begin{abstract}
Background: Poor dietary adherence to post-bariatric eating guidelines and associated maladaptive eating behaviors predict suboptimal post-bariatric weight loss outcomes. Psychometricallyevaluated measures that reliably detect such maladaptive behaviors are lacking.

Objectives: To describe the Dietary-adherence Intake and Eating Test (DIET), a brief self-report measure to detect post-bariatric maladaptive eating behaviors that have been previously associated with suboptimal weight loss post-bariatric surgery.

Methods: Preliminary psychometrics properties, including tempora stability, were examined in 109 patients within their first year post bariatric surgery.

Results: The 13-item measure demonstrated good test-retest reliability $(p<0.001)$, internal consistency $(a=0.86)$, and factor analysis results suggested all items load onto a single component solution.

Conclusion: The DIET is a brief, reliable, and internally consistent self-report measure with good psychometric properties that assesses the presence and frequency of a range of maladaptive post-bariatric eating behaviors. The DIET fills an important gap in the literature as it is, to our knowledge, the first psychometrically-valid questionnaire to assess the range of maladaptive eating behaviors shown by previous research to be associated with greater risks of suboptimal post-bariatric weight loss. The DIET, which can be easily administered post-bariatric surgery, possesses valuable clinical utility in that the maladaptive eating behaviors it captures can serve as the target of early, postsurgical intervention, thus potentially improving post-bariatric surgery weight loss outcomes.
\end{abstract}

\section{Abbreviations}

DIET: Dietary-adherence Intake and Eating Test; LOC: Loss of Control; EFA: Exploratory Factor Analysis; PA: Horn's Parallel Analysis; MAP: Velicer's Minimum Average Partial

\section{Introduction}

Bariatric surgery is the most effective treatment for morbid obesity [1]. However, while approximately $70 \%$ of patients achieve successful outcomes, up to $30 \%$ do not [1]. Post-surgical weight loss failure (commonly defined as a loss of less than $50 \%$ of one's excess initial weight [2], has been associated with lessened improvements in

\section{Journal of \\ Obesity and Bariatrics}

Alison M Darcy, Athena H Robinson, Sarah Adler,
Sarah Pajarito, Lilya Osipov and Debra L Safer*

Department of Psychiatry and Behavioral Sciences, Stanford University School of Medicine, Stanford, California, USA

\section{*Address for Correspondence}

Debra L. Safer, MD, Department of Psychiatry and Behavioral Sciences, Stanford, University School of Medicine, 401 Quarry Road, Stanford, California, USA, Tel: +1(650)723-7928; E-mail: dlsafer@stanford.edu

Copyright: (c) 2015 Darcy AM, et al. This is an open access article distributed under the Creative Commons Attribution License, which permits unrestricted use, distribution, and reproduction in any medium, provided the original work is properly cited.

Submission: 27 January 2015

Accepted: 09 February 2015

Published: 13 February 2015

Reviewed \& Approved by: Dr. Hope Landrine, Director of the Center for Health Disparities, East Carolina University, USA

psychiatric and medical comorbidities [1]. Suboptimal weight losses from poor initial weight loss and/or weight regain tend to occur by 24 months post-bariatric surgery [3].

From a clinical standpoint, intervening at the point when suboptimal weight outcomes have been observed (i.e., by 24 months post-bariatric surgery) may be too late; by that point maladaptive post-bariatric eating behaviors may already be engrained. Stated differently, waiting too long to intervene may miss a critical period for intervention during which patients may more readily adopt, practice, and sustain healthful post-bariatric eating behaviors. Thus, a psychometrically valid measure that detects early onset of maladaptive post-bariatric eating behaviors associated with sub-optimal outcomes is necessary to identify patients in need of targeted interventions that address these behaviors and promote optimal weight loss. In addition, such a measure could be used as part of longitudinal studies to further assess the short- and longer-term relationship between maladaptive post-bariatric eating behaviors and suboptimal weight outcomes.

Increasing evidence demonstrates that poor adherence to the recommended post-bariatric dietary guidelines [4] and associated maladaptive post-bariatric eating behaviors (i.e., loss of control over eating) [5] are robust predictors of suboptimal weight-loss outcomes. Although such behaviors have been previously studied, their measurement has been problematic. A recent systematic review identified 35 self-report measures that assess disordered eating following bariatric surgery. Of these, only $20 \%$ included psychometric evaluation within post-bariatric samples [6], and almost exclusively included assessments originally developed using a traditional eating disorder theoretical framework intended for patients with primary eating disorders. One significant limitation of such assessments, even when modified for the post-bariatric population, is their focus on determining whether loss of control (LOC) and/or distorted weight or shape concerns are present. This theoretical focus fails to 1) acknowledge that LOC and/or distorted weight and shape concerns may manifest differently within the bariatric population and thus require nuanced definitions within this sub-group or 2) detect maladaptive eating behaviors that may or may not be accompanied by LOC or distorted weight or shape concerns, yet are highly relevant 
Citation: Darcy AM, Robinson AH, Adler S, Pajarito S, Osipov L, et al. Psychometric Evaluation of the Dietary-Adherence Intake and Eating Test (DIET): A Measure for Early Detection of Maladaptive Eating Behaviors after Bariatric Surgery. J Obes Bariatrics. 2015;2(1): 6.

to post-bariatric populations (i.e., grazing, or nibbling, snacking, or eating small amounts of food in an unplanned and repetitious way).

The other $80 \%$ of questionnaires that assess post-bariatric maladaptive eating behaviors were primarily developed for the purpose of single studies and did not include psychometric testing nor sufficient detail to enable evaluation, replication, or comparison with other measures [6]. For example, the WATCH is a risk assessment measure specifically developed to assess for post-bariatric behaviors associated with increased risk for post-bariatric suboptimal weight loss outcomes. It includes questions such as, "Are you having a hard time consistently adhering to the recommendations of your surgery team"? [7]. Although this measure appears to possess good face validity, its reliability is unclear given the absence of psychometric evaluation. Moreover, the WATCH fails to quantify the frequency in which a patient engages in the maladaptive behaviors, thereby missing practical and informative data about the manifestation of such behaviors.

Only one assessment questionnaire was found that was specifically developed for and psychometrically evaluated among a post-bariatric sample: the Dutch Sweet Eating Questionnaire [8]. While this questionnaire quantifies the frequency at which individuals engage in sweet eating, its utility is limited because only behaviors directly related to sweet eating are assessed.

Thus, there is a need for a reliable self-report questionnaire to assess for the presence and frequency of a range of maladaptive postbariatric eating behaviors that have been previously associated with increased risk of suboptimal weight outcomes. The current study describes the systematic development and psychometric properties of a new measure, the Dietary-adherence Intake and Eating Test (DIET) The DIET assesses for the presence of maladaptive eating behaviors that have the most empirical support as negative psychosocial predictors of suboptimal weight loss outcome in bariatric surgery $[9,10]$.

\section{Methods}

\section{Participants and recruitment}

Participants were recruited at their 2-week pre-bariatric visit, after providing informed consent. One hundred and nine participants completed the DIET at their regularly scheduled followup appointment at the bariatric clinic (2-weeks pre-bariatric surgery and at 6 weeks, 3 months, 6 months, and 12 months post-bariatric surgery appointments).

For test-retest analysis to examine temporal stability, participants were contacted via e-mail 2 to 4 weeks after completing the initial DIET and asked to complete a second DIET. Approximately half $(n=53)$ of the original 109 completed the second questionnaire.

\section{Measures}

The following demographic characteristics were collected via selfreport: gender, age, type of surgery; date of surgery; height; current weight and pre-surgical weight.

Development of the DIET: Questionnaire items for the DIET (see Table 1) were generated from several sources. First, we included items identified by an earlier research conducted by our group using a separate dataset [10]. In that study, 84 pre- and postbariatric variables were identified, through an extensive review of the literature, as factors associated with an increased likelihood for suboptimal post-bariatric weight loss outcomes. Signal detection analysis then identified the most sensitive and specific predictors of suboptimal weight loss outcomes among 274 post-bariatric patients. The results are described elsewhere [10]. Briefly, the variable most strongly associated with suboptimal weight outcomes was selfreported adherence to the recommended post-bariatric dietary guidelines, followed by the frequency of post-bariatric grazing. Both of these variables were therefore included as DIET items.

Additional DIET items came from previous studies of factors found to be moderately correlated with post-bariatric dietary adherence (i.e., mindless eating, emotional eating). Such factors also help provide a more granular description of the global construct of dietary adherence. To detect disordered eating, the DIET included items assessing binge eating and loss of control for those episodes since the latter has been demonstrated to have greater predictive value [5]. For clarity and consistency, the most commonly cited definitions available in the literature for maladaptive eating behaviors were used.

Third, to increase item relevance for patients across different phases of post-surgical physiological capacity and corresponding dietary guidelines, which varies by time lapse since surgery (i.e., liquid diet for the first 2 weeks, etc.), the DIET includes items assessing the presence and degree of urges to engage in maladaptive eating behaviors. This allows for the detection of the potential for disordered behaviors even when individuals are physiologically limited in the amount of food they can eat.

To address gaps in the extant literature around quantitative assessment of maladaptive post-bariatric eating behaviors, the DIET assessed the frequency of these behaviors in number of days (0-7) individuals engaged in the behavior over the past week. Unlike the Eating Disorders Examination [11], that dichotomously assesses the presence or absence of LOC, the DIET includes a quantitative description of the degree of LOC (on a 0-6 scale). Dietary adherence was assessed via a 9-point response scale to maintain consistency with findings from previous literature [4].

Examination using the Flesch-Kincaid Grade Level demonstrated that the DIET items are at a 9th Grade Level (Flesch-Kincaid Grade Level $=9.2$; Flesch Reading $=61.5$ ) .

\section{Data analysis}

Maximum likelihood exploratory factor analysis (EFA) was conducted to identify latent constructs. Horn's parallel analysis (PA) [12] and Velicer's minimum average partial (MAP) [13] analyses were used to determine the number of factors to extract. These methods were chosen over more conventional approaches (eg. Kaiser's eigen value $>1$ rule, Cattell's scree plot test) given evidence suggesting the tendency of the more traditional approaches to overestimate or inconsistently identify the number of factors [13]. Syntax specified by O'Connor [14] was used to identify the number of factors. Briefly, the PA generates a random set of the same number of observations and variables as in the observed dataset. Using principal components analysis, eigen values are extracted from the random data, and this extraction is repeated for 100 iterations. Eigen values from the 95th 
Citation: Darcy AM, Robinson AH, Adler S, Pajarito S, Osipov L, et al. Psychometric Evaluation of the Dietary-Adherence Intake and Eating Test (DIET): A Measure for Early Detection of Maladaptive Eating Behaviors after Bariatric Surgery. J Obes Bariatrics. 2015;2(1): 6.

ISSN: $2377-9284$

Table 1: Dietary Intake and Eating habits Test (DIET).

\begin{tabular}{|c|c|c|}
\hline \multicolumn{2}{|c|}{ Patient ID Number } & \\
\hline Current weight & Ibs & \\
\hline Current height & feet & inches \\
\hline Gender & female __ male, & \\
\hline Age & years & \\
\hline \multicolumn{3}{|l|}{ Month of surgery } \\
\hline \multicolumn{3}{|l|}{ Year of surgery } \\
\hline Weight immediately prior to surgery & Ibs & $\begin{array}{l}\text { Highest Weight Ever } \\
\text { lbs }\end{array}$ \\
\hline Type of surgery: & gastric lap band gastric sleeve gastric bypass & other, specify \\
\hline
\end{tabular}

To the best of your ability, please answer the following questions about what has been true for you for the OVER THE PAST 7 DAYS.

Over the past 7 days...

\begin{tabular}{|c|c|c|c|c|c|c|c|c|}
\hline & 0 Days & 1 Day & 2 Days & 3 Days & $\begin{array}{c}4 \\
\text { Days }\end{array}$ & $\begin{array}{c}5 \\
\text { Days }\end{array}$ & $\begin{array}{c}6 \\
\text { Days }\end{array}$ & $\begin{array}{c}7 \\
\text { Days }\end{array}$ \\
\hline $\begin{array}{l}\text { 2. ...on how many days did you graze (e.g., nibbling, snacking, or } \\
\text { eating small amounts of food in an unplanned and repetitious way)? }\end{array}$ & $\square$ & $\square$ & $\square$ & $\square$ & $\square$ & $\square$ & $\square$ & $\square$ \\
\hline $\begin{array}{l}\text { 4. ...on how many days did you eat until your plate was clean } \\
\text { (regardless of whether you were hungry or full)? }\end{array}$ & $\square$ & $\square$ & $\square$ & $\square$ & $\square$ & $\square$ & $\square$ & $\square$ \\
\hline $\begin{array}{l}5 . \quad \text {...on how many days did you continue to eat, even though you } \\
\text { felt physically full? }\end{array}$ & $\square$ & $\square$ & $\square$ & $\square$ & $\square$ & $\square$ & $\square$ & $\square$ \\
\hline $\begin{array}{l}\text { 6. ...on how many days did you eat until you were overly full, } \\
\text { beyond comfort? }\end{array}$ & $\square$ & $\square$ & $\square$ & $\square$ & $\square$ & $\square$ & $\square$ & $\square$ \\
\hline $\begin{array}{l}7 . . . \text { on how many days did you experience emotional eating (defined } \\
\text { as not being able to resist eating in response to emotions or emotional } \\
\text { arousal)? }\end{array}$ & $\square$ & $\square$ & $\square$ & $\square$ & $\square$ & $\square$ & $\square$ & $\square$ \\
\hline
\end{tabular}

To the best of your ability, please answer the following questions about what has been true for you for the OVER THE PAST 7 DAYS.

\section{Over the past 7 days...}

1. ...how well did you follow the diet plan given to you by the dietician/nutritionist/surgeon?

\begin{tabular}{|c|c|c|c|c|c|c|c|c|}
\hline 1. ...how well did you follow the diet plan given to you by the dietician/nutritionist/surgeon? & 5 & 6 & 7 \\
\hline 1 & 2 & 3 & 4 & Moderately well & & 9 \\
\hline Not well at all & & & & Very well \\
\hline$\square$ & $\square$ & $\square$ & $\square$ & $\square$ & $\square$ \\
\hline
\end{tabular}

8. Over the past 7 days, how often did you experience loss of control eating (e.g., you couldn't resist starting to eat or stop once you began)? (Note: The total amount of food could be of any amount, very small to vary large)

\begin{tabular}{|c|c|c|c|c|c|c|c|}
\hline $\begin{array}{c}0 \\
\text { days }\end{array}$ & 1 & 2 & 3 & 4 & 7 \\
days & & 5 \\
\hline
\end{tabular}

10. Over the past 7 days, how often did you experience binge eating (e.g., eat an amount of food that others would agree was excessive while simultaneously experiencing a loss of control)?

\begin{tabular}{|c|c|c|c|c|c|c|c|c|}
\hline $\begin{array}{c}0 \\
\text { days }\end{array}$ & 1 & 2 & 3 & 4 & 7 \\
days & & 5 \\
\hline \\
\hline
\end{tabular}


Citation: Darcy AM, Robinson AH, Adler S, Pajarito S, Osipov L, et al. Psychometric Evaluation of the Dietary-Adherence Intake and Eating Test (DIET): A Measure for Early Detection of Maladaptive Eating Behaviors after Bariatric Surgery. J Obes Bariatrics. 2015;2(1): 6.

ISSN: $2377-9284$

12. Over the past 7 days, how often did you have urges to eat off the plan given to you by dietician/nutritionist/surgeon?

\begin{tabular}{|c|c|c|c|c|c|c|c|}
\hline $\begin{array}{c}0 \\
\text { days } \\
\square\end{array}$ & $\begin{array}{l}1 \\
\square\end{array}$ & $\begin{array}{l}2 \\
\square\end{array}$ & $\begin{array}{l}3 \\
\square\end{array}$ & $\begin{array}{c}4 \\
\text { days } \\
\square\end{array}$ & $\begin{array}{l}5 \\
\square\end{array}$ & $\begin{array}{l}6 \\
\square\end{array}$ & $\begin{array}{c}7 \\
\text { days } \\
\square\end{array}$ \\
\hline \multicolumn{8}{|c|}{ 13. On average, on those days (from question 12), how strong were the urges? If no urges to eat off plan, please rate "0" } \\
\hline & $\begin{array}{c}0 \\
\text { Not at all strong }\end{array}$ & 1 & $\begin{array}{c}2 \\
\text { Moderately strong }\end{array}$ & 3 & $\begin{array}{c}4 \\
\text { Definitely strong }\end{array}$ & 5 & $\begin{array}{c}6 \\
\text { Extremely strong }\end{array}$ \\
\hline & $\square$ & $\square$ & $\square$ & $\square$ & $\square$ & $\square$ & $\square$ \\
\hline
\end{tabular}

percentile of the set are then compared to those from the observed data, and those with values greater than the random data are retained. Principal axis factoring was chosen over principal components analysis because the aim was to identify the latent structure of the data rather than reduce the number of variables. All EFA analyses were conducted in SPSS version 22.0. We used a cut-off of 0.4 to guide factor loading. Total score for the DIET is derived by summing all item scores (with the dietary adherence item being reverse scored) and dividing by the total number of items. Test-retest was conducted on all individuals who provided data on two occasions using Pearson $r$

\section{Ethical approval}

All procedures performed in studies involving human participants were in accordance with the ethical standards of the institutional and/or national research committee and with the 1964 Helsinki declaration and its later amendments or comparable ethical standards.

\section{Informed consent}

Informed consent was obtained from all individual participants included in the study. The protocol was approved by Stanford's Institutional Review Board.

\section{Results}

\section{Participant characteristics}

Data were analyzed from a total of 109 individuals with a mean age of $46.70(\mathrm{SD}=11.75)$ and mean BMI of $39.54(\mathrm{SD}=9.14)$. Eightyfour were female $(77.1 \%)$ and $25(22.9 \%)$ were male. In terms of race/ethnicity, $49.5 \%$ were Caucasian, $36.4 \%$ were Latino/Hispanic, 3\% were Asian, 3\% were Native American or Alaskan Native, $2 \%$ were African American, and 6.1\% reported more than one race. With regard to level of education, $6 \%$ had not graduated from high school, $22 \%$ were high school graduates, $26 \%$ had some college, $18 \%$ had graduated from a 2 -year college, $20 \%$ were graduates from a 4 -year college, $1 \%$ had some post-college education, and $7 \%$ held graduate or professional degrees. Surgery types were gastric sleeve $(n=51 ; 46.8 \%)$, gastric bypass $(n=42 ; 38.5 \%)$, and gastric lap band $(n=15 ; 13.8 \%)$. One individual (0.09\%) reported "other" for type of bariatric surgery. Participants had surgery on average $3.63(\mathrm{SD}=3.12)$ months prior to completion of the DIET.

\section{Temporal stability and internal reliability}

The test-retest analysis on 53 patients revealed a significant correlation between DIET score at time 1 and time $2(\mathrm{r}(53)=0.532$; $\mathrm{p}<0.001)$. The DIET has a Cronbach's alpha of 0.79 .

\section{Factor analysis}

Both the PA and MAP suggested that a single component should be extracted; thus the solution was not rotated. As can be seen in Table 2, 12 out of the 13 items loaded adequately onto a single factor, with $41.99 \%$ of the variance accounted for. Since the Eating beyond Comfort item failed to load adequately, it was removed. The analysis was repeated and resulted in a 12 -item single component solution, accounting for $45.46 \%$ of the variance. Table 3 provides normative values on the total score of the measure with these 12 items.

\section{Conclusion}

This study explored the psychometric properties of the DIET, a brief self-report measure designed to detect the presence and frequency of maladaptive post-bariatric eating behaviors. The maladaptive eating behavior items included in this measure have the greatest empirical support for their association with risk of suboptimal post-bariatric weight loss outcomes (see recent review by Sheets et al. [9]. The 13-item measure was piloted among postbariatric patients between 2 weeks and 12 months after surgery and demonstrated good temporal stability and internal reliability, with 12 out of 13 items loading onto a single construct.

The one item that did not load adequately onto the single construct inquired about eating beyond comfort. However, because endorsement of this item was so rare in the current sample, it is possible that detection was due to insufficient variance. It is noteworthy that once this item was removed, the resulting DIET remained psychometrically robust and internally consistent for patients at different time points from surgery and through the 12 months follow-up assessment. This is meaningful given the changing dietary restrictions physiologically imposed at different post-bariatric phases, indicating that the DIET is appropriate for use both among early and later phase post-bariatric surgery patients.

There are several benefits of the DIET for both clinical and research purposes. First, unlike post-surgical measures adapted from the eating disorder field, the DIET does not follow a purely eating disorder conceptual framework [6]. Therefore, the DIET does not require a distinction between maladaptive eating behaviors due to underlying psychological causes versus underlying physiological causes (i.e., whether grazing is driven by eating, shape and weight concerns or by a desire to avoid and/or relieve physical symptoms related to post-surgical changes), a distinction which previously posed a major challenge to the development of valid and reliable measures among the bariatric population [6]. Second, the DIET was developed specifically using data from a post-bariatric population, unlike previous measures which were developed based on other populations and subsequently adapted for bariatric populations [6]. 
Citation: Darcy AM, Robinson AH, Adler S, Pajarito S, Osipov L, et al. Psychometric Evaluation of the Dietary-Adherence Intake and Eating Test (DIET): A Measure for Early Detection of Maladaptive Eating Behaviors after Bariatric Surgery. J Obes Bariatrics. 2015;2(1): 6.

ISSN: $2377-9284$

Table 2: Single component item loadings on the DIET for the final 12-item solution.

\begin{tabular}{|c|c|}
\hline Item & Loading \\
\hline $\begin{array}{l}\text { Over the past week ( } 7 \text { days) on how many days did you experience grazing (e.g., nibbling, snacking, or eating small amounts of food in an } \\
\text { unplanned and repetitious way)? }\end{array}$ & 0.632 \\
\hline $\begin{array}{l}\text { Over the past week ( } 7 \text { days) on how many days did you experience mindless eating (defined as eating while watching TV or doing other activities } \\
\text { such as eating at the computer, in the car, at work, etc.)? }\end{array}$ & 0.64 \\
\hline Over the past week ( 7 days) ... on how many days did you eat until your plate was clean (regardless of whether you were hungry or full)? & 0.424 \\
\hline Over the past week (7 days) on how many days did you continue to eat, even though you felt physically full? & 0.576 \\
\hline $\begin{array}{l}\text { Over the past week ( } 7 \text { days) ... on how many days did you experience emotional eating } \\
\text { (defined as not being able to resist eating in response to emotions or emotional arousal)? Italics }\end{array}$ & 0.881 \\
\hline $\begin{array}{l}\text { Over the past week ( } 7 \text { days) ... on how many days did you experienced loss of control eating (e.g., experiencing that you couldn't resist starting to } \\
\text { eat or stop once you began)? (Note: The total amount of food could be of any amount, very small to very large.) }\end{array}$ & 0.888 \\
\hline On those days, the degree $(0-6)$ to which you experienced a sense of loss of control while eating, on average, was & 0.851 \\
\hline $\begin{array}{l}\text { Over the past week ( } 7 \text { days) ... on how many days did you binge eat (e.g., eat an amount of food that others would agree was excessive while } \\
\text { simultaneously experiencing a loss of control)? }\end{array}$ & 0.643 \\
\hline Over the past 7 days (0-7), how often did you have urges to eat off the plan given to you by dietician/nutritionist/surgeon? & 0.533 \\
\hline On average, on those days, how strong were the urges $(0-6) ?$ & 0.699 \\
\hline How well (1-9) are you following the diet plan given to you by your surgeon/dietician/nutritionist? $(1=$ not at all well, $9=$ Very well. Reverse scored $)$ & 0.553 \\
\hline Eigenvalue & 5.45 \\
\hline$\%$ Variance accounted for & 45.46 \\
\hline
\end{tabular}

${ }^{1}$ The italicized item in parentheses was removed due to poor loading

Table 3: Normative values for the DIET among $\mathrm{N}=109$ post-bariatric surgical patients.

\begin{tabular}{|c|c|}
\hline Percentile & DIET score \\
\hline 5 & 0.08 \\
\hline 10 & 0.17 \\
\hline 15 & 0.25 \\
\hline 20 & 0.33 \\
\hline 25 & 0.38 \\
\hline 30 & 0.42 \\
\hline 35 & 0.50 \\
\hline 40 & 0.58 \\
\hline 45 & 0.67 \\
\hline 50 & 0.80 \\
\hline 55 & 0.92 \\
\hline 60 & 1.00 \\
\hline 65 & 1.13 \\
\hline 70 & 1.20 \\
\hline 75 & 1.41 \\
\hline 80 & 1.50 \\
\hline 85 & 1.92 \\
\hline 90 & 2.25 \\
\hline 95 & 2.79 \\
\hline 99 & 5.21 \\
\hline
\end{tabular}

Moreover, the DIET is brief, which limits the cognitive and time burden upon patients and scoring burden upon administrators. It is also easily administered (via either paper-and-pen or internetsurvey) and readily interpretable. Indeed, it was logistically practical to email the DIET survey link to patients who did not follow-up at their in-person post-bariatric clinic appointments, an unfortunate commonality among this population [15].

Limitations of this study are that neither predictive nor congruent validity of the DIET were measured. Indeed, longitudinal studies are needed to test the predictive validity of the DIET against an objective measure, such as post-bariatric weight outcomes obtained at least 24 months post-surgery. These forms of validity should be addressed in future studies.

In sum, the DIET fills an important gap in the literature. To our knowledge, it is the first brief psychometrically-valid selfreport questionnaire designed to detect and quantify the frequency of a range of maladaptive post-bariatric eating behaviors that have been previously associated with increased risk of suboptimal weight outcomes. The present study detailed the DIET's systematic and empirically-driven development, and found it to have good temporal stability and internal reliability. The DIET has valuable clinical utility in that the maladaptive eating behaviors it captures can serve as the targets of early, post-surgical behavioral interventions that may improve post-bariatric weight loss outcomes. In addition, use of the DIET in post-bariatric research studies would allow for standardization of post-bariatric maladaptive eating behaviors and therefore more accurate cross-study comparisons.

\section{References}

1. Sjöström L, Lindroos AK, Peltonen M, Torgerson J, Bouchard C, et al. (2004) Lifestyle, diabetes, and cardiovascular risk factors 10 years after bariatric surgery. N Engl J Med 351: 2683-2693. 
Citation: Darcy AM, Robinson AH, Adler S, Pajarito S, Osipov L, et al. Psychometric Evaluation of the Dietary-Adherence Intake and Eating Test (DIET): A Measure for Early Detection of Maladaptive Eating Behaviors after Bariatric Surgery. J Obes Bariatrics. 2015;2(1): 6.

ISSN: $2377-9284$

2. Livhits M, Mercado C, Yermilov I, Parikh JA, Dutson E, et al. (2010) Behaviora factors associated with successful weight loss after gastric bypass. Am Surg 76: $1139-1142$

3. Magro DO, Geloneze B, Delfini R, Pareja BC, Callejas F, et al. (2008) Longterm weight regain after gastric bypass: a 5 -year prospective study. Obes Surg 18: 648-651.

4. Sarwer DB, Wadden TA, Moore RH, Baker AW, Gibbons LM, et al. (2008) Preoperative eating behavior, postoperative dietary adherence, and weight loss after gastric bypass surgery. Surg Obes Relat Dis 4: 640-646.

5. White MA, Kalarchian MA, Masheb RM, Marcus MD, Grilo CM (2010) Loss of control over eating predicts outcomes in bariatric surgery: a prospective 24-month follow-up study. J Clin Psychiatry 71: 175-184.

6. Parker K, O'Brien P, Brennan L (2014) Measurement of disordered eating following bariatric surgery: a systematic review of the literature. Obes Surg 24: $945-953$

7. Coughlin JW, Guarda AS, Clark JM, Furtado MM, Steele KE, et al. (2013) A screening tool to assess and manage behavioral risk in the postoperative bariatric surgery patient: The WATCH. J Clin Psychol Med Settings 20: 456463.

8. van den Heuvel M, Hörchner R, Wijtsma A, Bourhim N, Willemsen D, et al (2011) Sweet eating: a definition and the development of the dutch sweet eating questionnaire. Obes Surg 21: 714-721.
9. Sheets CS, Peat CM, Berg KC, White EK, Bocchieri-Ricciardi L, et al. (2015) Post-operative psychosocial predictors of outcome in bariatric surgery. Obes Surg 25: 330-345.

10. Robinson AH, Adler S, Stevens HB, Darcy AM, Morton JM, et al. (2014) What variables are associated with successful weight loss outcomes for bariatric surgery after 1 year? Surg Obes Relat Dis 10: 697-704.

11. Fairburn CG, Cooper Z, O'Connor M (2008) Cognitive behaviour therapy and eating disorders. New York, NY: Guildford Press.

12. Velicer WF (1976) Determining the number of components from the matrix of partial correlations. Psychometrika 41: 321-327.

13. Hayton JC, Allen DG, Scarpello V (2004) Factor retention decisions in exploratory factor analysis: a tutorial on parallel analysis. Organ Res Methods 7: $191-205$

14. O'Connor BP (2000) SPSS and SAS programs for determining the number of components using parallel analysis and velicer's MAP test. Behav Res Methods Instrum Comput 32: 396-402.

15. Moroshko I, Brennan L, O'Brien P (2012) Predictors of attrition in bariatric aftercare: a systematic review of the literature. Obes Surg 22: 1640-1647.

\section{Acknowledgements}

This research was funded in part by a grant from the National Institute of Mental Health K23MH085732 awarded to Athena Robinson, PhD. 